\title{
Validación de la versión española del Cuestionario Benign Prostatic Hyperplasia Impact Index. "Estudio Validart"
}

\author{
Carballido Rodríguez $\mathrm{J}^{1}$, Grunfeld Abellán A², Escudero Callen A ${ }^{3}$, Gil Bermejo FJ ${ }^{4}$, \\ Regadera-Anechina $\mathrm{L}^{5}$, Badía Llach $\mathrm{X}^{6}$. \\ ${ }^{1}$ Servicio Urología. Hospital Universitario Clinica Puerta del Hierro. ${ }^{2}$ Ambulatorio de Moratalaz. Madrid. \\ ${ }^{3}$ Servicio Urología. Hospital Santiago Apóstol. Vitoria. ${ }^{4}$ Servicio Urología. Hospital Txagorritxu. Vitoria. \\ ${ }^{5}$ Departamento Médico. GlaxoSmithKline S.A. Tres Cantos (Madrid) \\ ${ }^{6}$ Health Economics \& Outcomes Research. IMS Health. Barcelona.
}

Actas Urol Esp. 2008;32(2):230-239

\section{RESUMEN}

VALIDACIÓN DE LA VERSIÓN ESPAÑOLA DEL CUESTIONARIO BENIGN PROSTATIC HYPERPLASIA IMPACT INDEX. "ESTUDIO VALIDART"

Introducción: La Hiperplasia Benigna de Próstata (HBP) es una enfermedad crónica que afecta la calidad de vida relacionada con la salud (CVRS). En España no hay cuestionarios específicos para evaluar la CVRS en esta enfermedad.

El objetivo del presente estudio fue validar la versión española del cuestionario Benign Prostatic Hyperplasia Impact Index (BII).

Material y métodos: Participaron hombres mayores de 49 años diagnosticados de HBP evaluados en tres visitas. Se analizaron las propiedades psicométricas del cuestionario en términos de validez, fiabilidad y sensibilidad al cambio.

Resultados: Se evaluaron 1.719 hombres con edad media (DE) de 68,36 años $(8,07)$ clasificados según gravedad de la HBP.

La validez longitudinal y la validez de constructo $(r>0,6)$ del cuestionario BII fueron moderadas-altas.

La consistencia interna $(\alpha=0,91)$, la fiabilidad test-retest $(\mathrm{CCI}=0,8)$ y la sensibilidad al cambio (tamaño efecto $=0,6)$ fueron buenas.

La CVRS mejoró significativamente durante el seguimiento $(\mathrm{p}<0,01)$.

Discusión: Hasta ahora no existían cuestionarios específicos de calidad de vida para población española con HBP. La versión española del cuestionario BII cumple las propiedades psicométricas necesarias para evaluar la CVRS en la HBP; confirmando además, el significativo impacto de la enfermedad en la calidad de vida de estos pacientes.

Palabras clave: Hiperplasia prostática. Calidad de vida. Estudios de validación. Cuestionarios.

\section{ABSTRACT}

VALIDATION OF THE SPANISH VERSION OF THE BENIGN PROSTATIC HYPERPLASIA IMPACT INDEX QUESTIONNAIRE. "VALIDART STUDY"

Introduction: Benign Prostatic Hyperplasia is a chronic disease that affects Health related quality of life (HRQL). In Spain there are no specific questionnaires to assess HRQL in patients suffering from BPH.

The objective of this study was to validate the Spanish version of the Benign Prostatic Hyperplasia Impact Index (BII) questionnaire.

Material and methods: Study participants were men over 49 with confirmed BPH diagnosis and evaluated in three visits.

Psychometric properties of the questionnaire were analyzed in terms of validity, reliability and sensitivity to change.

Results: A total of 1,719 men with mean (SD) age 68.36 were evaluated and classified according to the BPH severity.

Both longitudinal and construct validity $(r>0.6)$ of the BII questionnaire were moderate-high. Internal consistency $(\alpha=0.91)$, test-retest reliability $(\mathrm{CCI}=0.8)$ and sensitivity to change (effect size $=0.6)$ showed good results.

The HRQL improved significantly during the monitoring period $(\mathrm{p}<0.01)$.

Discussion: There were no available specific HRQL questionnaires for Spanish population with BPH until now. The Spanish version of the BII questionnaire fulfils with the psychometric properties that are necessary to evaluate HRQL in patients with BPH; furthermore, it has been confirm the considerable impact of this disease in the quality of life of this patients.

Keywords: Prostatic hyperplasia. Quality of life. Validation studies. Questionnaires. 
$\mathrm{L}$ a Hiperplasia Benigna de Próstata (HBP) es una enfermedad crónica y progresiva, con periodos sintomáticos de intensidad variable y una alteración de la calidad de vida, afectando tanto a las actividades cotidianas como a las relaciones personales ${ }^{1,2}$.

Esta patología se caracteriza por tener una alta prevalencia en edades avanzadas, con valores de hasta el $70 \%$ a partir de los 60 años ${ }^{3}$ y una elevada comorbilidad, asociándose a menudo con disfunción eréctil ${ }^{4}$, retención urinaria y enfermedades del tracto urinario ${ }^{5}$.

La HBP es una de las enfermedades que, pese a no conllevar riesgo de muerte, con más frecuencia afecta la Calidad de Vida Relacionada con la Salud (CVRS) de los hombres de edad avanzada ${ }^{3,6,7}$, esta repercusión es mayor cuanto más grave es la sintomatología urinaria ${ }^{8,9}$.

En la evaluación de una enfermedad, sobre todo si ésta es crónica, cada vez cobra más importancia el uso de medidas centradas en el paciente ${ }^{10}$. Analizar el estado de salud de los pacientes y su CVRS es esencial para la evaluación y manejo de la enfermedad en la práctica clínica $^{11}$.

En la $5^{\text {a }}$ Conferencia Internacional sobre HBP celebrada en París ${ }^{12}$, los expertos recomendaban realizar una evaluación global de los pacientes, empezando por la cuantificación de los sintomas y un examen físico y completándola con medidas centradas en el paciente. Para la cuantificación de los sintomas el instrumento por excelencia entre los investigadores y clínicos es el I-PSS y como medidas centradas en el paciente, el ítem Bother Store junto con otros instrumentos de calidad de vida específicos y validados.

En muchas ocasiones, la CVRS y el estado de salud en los pacientes con HBP han sido evaluados mediante cuestionarios genéricos como el EUROQoL-5D ${ }^{13}$, aunque para poder evaluar la CVRS de manera más integral, son necesarios instrumentos específicos que estén validados en el país de origen de la población y que sean cuestionarios breves para asegurar la factibilidad de su aplicación.

Así, los cuestionarios específicos más utilizados, entre otros, han sido el International Prostate Symptom Score (I-PSS) ${ }^{14,15}$, con dos dimensiones (sintomas y malestar por los mismos) y que tan solo incluye una pregunta de calidad de vida (item Bother Score), el cuestionario Danish Prostate Symptom Score (DAN-PSS-1) ${ }^{9,16}$, con dos dimensiones (sintomas y función sexual) y que sirve para la HBP pero que en realidad es específico de sintomas urinarios del tracto inferior y finalmente el Benign Prostatic Hyperplasia Impact Index (BII), desarrollado en EE.UU. y, al igual que los anteriores, traducido a varios idio$\operatorname{mas}^{17,18}$ y que sí es específico para la HBP.

Siguiendo las recomendaciones de París, para la evaluación de los sintomas, en el presente estudio se utilizó el I-PSS (con su ítem Bother Score como aproximación a la valoración del paciente), y para evaluar el impacto en la vida del paciente, se consideró importante poder utilizar un cuestionario que fuera específico para esta enfermedad como era el BII. Además, se consideró importante poder validar este cuestionario puesto que es el cuestionario específico para la HBP más breve y que abarca más dominios de la enfermedad, no solo los sintomas físicos, sino también preocupaciones y actividades cotidianas, aspectos más relacionados con la percepción subjetiva del individuo ${ }^{19}$.

Ambos instrumentos ya habian sido comparados en anteriores investigaciones sobre sintomas urinarios del tracto inferior, mostrando una clara asociación entre sus puntuaciones ${ }^{20}$.

Por este motivo, el objetivo principal de este estudio fue evaluar las propiedades de medición de la versión española del BII. Secundariamente se evaluó la calidad de vida de los pacientes a lo largo del estudio y según su gravedad.

\section{MATERIAL Y MÉTODOS}

\section{Diseño y población del estudio}

Se llevó a cabo un estudio epidemiológico, prospectivo y multicéntrico en el que se evaluaron pacientes varones mayores de 49 años diagnosticados de HBP que no presentaban otras enfermedades génito-urinarias y que no estaban participando en ningún estudio con medicamentos, situaciones ambas que podían enmascarar los resultados del presente estudio. Estos pacientes fueron incluidos en el estudio consecutivamente y clasificados, a partir de las puntuaciones obtenidas en el I-PSS, en tres grupos de gravedad: leves, moderados y graves. 
Los pacientes realizaron 3 visitas. El tiempo medio entre la visita 1 (basal) y las de seguimiento fue a la visita 2 de 2,85 meses y a la visita 3 de 6,34 meses.

En la visita basal se registraron variables sociodemográficas como la edad y el nivel de estudios; variables clínicas, como la fecha de diagnóstico de la HBP, el tratamiento actual para la enfermedad (indicando principio activo, dosis diaria y fecha de inicio) o las enfermedades concomitantes (respiratorias, dermatológicas, intestinales, metabólicas, alergias, colesterol y/o triglicéridos, hipertensión, cardiopatía, neoplasias, diabetes, etc...); y variables humanísticas (cuestionario BII, cuestionario I-PSS, con el item Bother Score, estado de salud general percibido por el propio paciente y valoración clínica del estado de salud del paciente por parte del médico).

La puntuación del I-PSS de esta visita se utilizó para clasificar al paciente en uno de los tres grupos de gravedad de la sintomatología anteriormente indicados.

En las visitas de seguimiento se recogieron motivos de abandono y posibles cambios en el tratamiento indicado en la visita anterior, se administraron de nuevo los cuestionarios BII, IPSS y el ítem Bother Score y se preguntó por el estado de salud percibido por el paciente y por la valoración clínica del estado de salud por parte del médico, respecto a la visita anterior.

\section{Medidas de calidad de vida relacionada con la salud}

El BII es un cuestionario específico para evaluar el impacto de los síntomas urinarios de la HBP en la calidad de vida y el estado de salud de los pacientes con esta enfermedad. Fue elaborado a partir de la revisión de cuestionarios genéricos y específicos ya publicados ${ }^{17}$. Consta de 4 itemes que exploran 4 áreas de la CVRS: malestar físico, preocupación por la salud, molestias e impedimento para realizar actividades cotidianas.

Todos los itemes tienen una escala de respuesta tipo Likert, los tres primeros con 4 opciones de respuesta y el último con 5 opciones de respuesta. La puntuación total oscila entre 0 (mejor CVRS) y 13 (peor CVRS) (Fig. 1).

El I-PSS es otro un cuestionario específico en urología muy empleado en investigación y en la práctica clínica y validado en población españo$1 \mathrm{a}^{21}$. Consta de 8 ítemes distribuidos en dos dimensiones: sintomas y preocupación por los síntomas con una escala de respuesta tipo Likert de 7 opciones. La puntuación oscila entre 0 (mejor CVRS) y 35 (peor CVRS). Según la puntuación total, se puede clasificar la sintomatologia de la HBP en tres grupos de gravedad: leve (0-7 puntos), moderada (8-19 puntos) y grave (20-35 puntos).

El último item (Bother Score) que puntúa aparte, mide la CVRS ${ }^{22}$.

\begin{tabular}{|c|c|c|c|c|c|}
\hline \multicolumn{6}{|c|}{ ÍNDICE DEL IMPACTO DE LA HIPERPLASIA BENIGNA DE PRÓSTATA (BPH-II) } \\
\hline & Nada & $\begin{array}{l}\text { Sólo un } \\
\text { poco }\end{array}$ & Algo & Mucho & \\
\hline $\begin{array}{l}\text { 1. Durante los últimos } 30 \text { días fisco le han causado } \\
\text { los poblemas urinarios }\end{array}$ & 0 & 1 & 2 & 3 & \\
\hline $\begin{array}{l}\text { 2. Durante los últimos } 30 \text { días, ¿cuánta preocupación por } \\
\text { su salud le han causado los problemas urinarios? }\end{array}$ & 0 & 1 & 2 & 3 & \\
\hline $\begin{array}{l}\text { 3. Durante los últimos } 30 \text { días, ¿en general cuánto le han } \\
\text { molestado los problemas urinarios? }\end{array}$ & 0 & 1 & 2 & 3 & \\
\hline \multirow{2}{*}{$\begin{array}{l}\text { 4. Durante los últimos } 30 \text { días, ¿con qué frecuencia los } \\
\text { problemas urinarios le han impedido hacer la clase de } \\
\text { cosas que hace normalmente? }\end{array}$} & 0 & 1 & 2 & 3 & 4 \\
\hline & Nunca & $\begin{array}{l}\text { Pocas } \\
\text { veces }\end{array}$ & $\begin{array}{l}\text { Algunas } \\
\text { veces }\end{array}$ & $\begin{array}{c}\text { Casi } \\
\text { siempre }\end{array}$ & Siempre \\
\hline
\end{tabular}

FIGURA 1. Cuestionario Benign Prostatic Hyperplasia Impact Index (BII). 


\section{Cálculo del tamaño muestral}

La predeterminación del tamaño muestral se calculó de forma que permitiera evaluar las propiedades psicométricas del cuestionario BII. Para poder evaluar la sensibilidad al cambio del cuestionario se tenían que poder detectar cambios en las puntuaciones a lo largo del estudio correspondientes a 0,20 desviaciones estándar, con un nivel de significación de 0,01 y un poder estadístico de 0,80.

Además, para que el BII resultara válido, debía presentar diferencias en las puntuaciones del cuestionario de al menos 0,2 desviaciones estándar (diferencia pequeña) entre los niveles de gravedad basal. Así pues, aceptando un riesgo $\alpha$ de 0,05 y un riego beta de 0,20, y asumiendo un riesgo del pérdidas a los largo del estudio del 20\%, se precisaba un tamaño muestral de 1.842 pacientes, distribuidos según gravedad en 307 leves, 1.228 moderados y 307 graves.

\section{Análisis estadistico}

Se realizó un análisis descriptivo de las características sociodemográficas y clinicas de la muestra. La descripción de la muestra se realizó según el nivel de gravedad de la sintomatología. Se compararon las características de los pacientes según los 3 niveles de gravedad utilizando para la edad, el tiempo de diagnóstico y la puntuación obtenida en el Bother Score la prueba ANOVA, y para el nivel de estudios, las enfermedades concomitantes y la prescripción de tratamiento la prueba chi-cuadrado.

\section{Propiedades psicométricas \\ Factibilidad}

Se analizó el porcentaje de pacientes que no respondieron a la totalidad del cuestionario BII.

\section{Validez}

La capacidad de un instrumento para medir el constructo que tiene que medir se evaluó en términos de validez transversal (validez de constructo), y de validez longitudinal.

La validez de constructo se determinó analizando la relación entre las puntuaciones del BII y las puntuaciones en el I-PSS, el estado de salud general valorado por el médico y por los propios pacientes, y el item independiente de calidad de vida (Bother Score). Los pacientes que presentaban una menor gravedad según el I-PSS debian obtener una menor puntuación en el cuestionario BII, es decir, los sintomas de la HBP debian tener un menor impacto en su calidad de vida. Del mismo modo, los pacientes que declaraban un peor estado de salud o una menor calidad de vida debian mostrar mayores puntuaciones en el cuestionario BII. Para analizar estas relaciones se utilizó el ANOVA y el coeficiente de correlación de Spearman. Para analizar la relación entre las puntuaciones del I-PSS y del BII se calculó el coeficiente de correlación de Pearson.

La validez longitudinal se evaluó a partir de la relación entre los cambios obtenidos en las puntuaciones del BII a los 6 meses y los cambios en las puntuaciones del I-PSS, el Bother Score y el estado de salud percibido por los pacientes. Para evaluar si los pacientes que presentaban un mayor cambio en el estado de salud, calidad de vida o sintomatología, también mostraban mayores cambios en las puntuaciones del cuestionario entre la visita inicial y a los 6 meses, se calculó el coeficiente de correlación de Spearman.

\section{Fiabilidad}

La consistencia interna se evaluó con el coeficiente $\alpha$ de Cronbach, valor que determina el grado de correlación entre los ítemes del cuestionario. Se definió una consistencia interna aceptable a partir de un coeficiente superior o igual a $0,7^{23}$. La fiabilidad test-retest, propiedad que determina la estabilidad temporal en las mediciones en condiciones similares (ante ausencia de cambios en la salud las puntuaciones no deben variar), se evaluó con el coeficiente de Correlación Intraclase (CCI) y que se define como la proporción de la variabilidad total que es debida a la variabilidad de los pacientes ${ }^{24}$. Un valor superior a 0,75 se considera un acuerdo excelente ${ }^{25}$. La fiabilidad test-retest se determinó entre la visita basal y a los 3 meses en aquellos pacientes que no declararon cambios en su estado de salud.

\section{Sensibilidad al cambio}

La capacidad del BII para reflejar cambios reales en la CVRS a lo largo del tiempo se evaluó calculando el tamaño del efecto, medida cuantitati- 
va que indica la importancia de un efecto. Según esto, se habla de "efecto pequeño" $(0,2-0,5)$, "efecto medio" $(0,6-0,8)$ y "efecto grande" $(>0,8)^{26}$. Este indice se ha calculado a partir de la diferencia entre la puntuación obtenida en el BII en la visita basal y a los 6 meses, dividida entre la desviación estándar de la visita basal y se calculó para cada una de las 6 categorias de cambio en el estado de salud declarado por el paciente, que oscilaban entre "mucho mejor" y "mucho peor".

Por último, se calculó la mínima diferencia clínicamente importante (MDCI), diferencia más pequeña en las puntuaciones del BII que el paciente percibe como beneficiosa. La MDCI es aquella puntuación que, en ausencia de efectos adversos o grandes costes, orientaría a un clínico para instaurar o modificar un tratamiento ${ }^{27}$. Se calculó la MCDI como la diferencia en las puntuaciones obtenidas en la visita basal y a los 6 meses entre los pacientes que declararon haber "mejorado moderadamente" su estado de salud.

\section{Calidad de Vida relacionada con la Salud (CVRS)}

Una vez confirmada la validez del cuestionario BII, se analizó la evolución de la calidad de vida en los pacientes con HBP durante los 6 meses de estudio. Se analizaron los cambios en la puntuación en cada visita de seguimiento respecto la visita basal, analizando si las puntuaciones evolucionaban de manera significativa mediante la prueba para datos apareados de Friedman.

\section{RESULTADOS}

La muestra teórica prevista era de 1.842 pacientes, debido a las bajas en el número final de investigadores participantes (278 de los 380 previsto), finalmente se incluyeron 1.737 pacientes con diagnóstico de HBP, de los cuales 1.719 resultaron evaluables. En algunas ocasiones no fue posible obtener una respuesta, por lo que la muestra final en cada una de las variables podía ser inferior a los 1.732 pacientes.

Del total de participantes evaluables, un 4,3\% (75) no finalizó el estudio a los 6 meses de seguimiento: el $1 \%$ (16) por pérdida de seguimiento, el $1,1 \%$ (19) por decisión del paciente, el $0,5 \%$ (8) por decisión del investigador y el 1,8\% (32) por otros motivos, algunos de los cuales fueron: fallecimiento $0,1 \%$, efectos secundarios o intolerancia farmacológica $0,5 \%$,intervenciones quirúrgicas relacionadas con la HBP 0,7\%.

En la Tabla 1 se presentan las caracteristicas sociodemográficas y clinicas basales de la población según gravedad medida a partir de las puntuaciones del I-PSS.

Se observaron diferencias estadísticamente significativas en la edad de los pacientes según la gravedad de los sintomas $(\mathrm{p}<0,01)$, presentando mayor gravedad los pacientes de más edad.

En cuanto al tratamiento, estaban tomando alguna medicación para la HBP el 49,6\% (118) de los pacientes con sintomatología leve, el $76,5 \%$ (729) de los pacientes con sintomatología moderada y el 78,7\% (409) de los pacientes con sintomatología grave $(\mathrm{p}<0,01)$, siendo los $\alpha$-bloqueantes los más prescritos en los dos últimos grupos.

Finalmente, el 77,3\% (1.328) de los pacientes presentaba alguna enfermedad concomitante. En los tres grupos de gravedad la presencia de alguna enfermedad fue similar. En algunas enfermedades concretas como la hipertensión, los pacientes más graves fueron los que más refirieron padecerla $(\mathrm{p}<0,01)$.

En cuanto a las variables centradas en el paciente, cabe destacar la buena concordancia (kappa $=0,68$; IC 95\%) entre el estado de salud percibido por los propios pacientes y la valoración que hacía el médico del mismo.

La gravedad de los pacientes según el cuestionario I-PSS, se registró en 1.719 casos. El 13,9\% (239) se clasificó como leve, el 55,7\% (957) como moderado y el $30,4 \%$ (523) como grave.

En la Tabla 2 se muestran las puntuaciones basales de los cuestionarios I-PSS, BII y del item Bother Score según la gravedad. En las tres pruebas, a mayor gravedad de la sintomatología de la HBP mayor puntuación en los cuestionarios, indicando un mayor impacto de los síntomas en la vida de los pacientes.

\section{Factibilidad}

El porcentaje de respuesta completa al cuestionario BII fue del 99,9\% (1.730). Tan sólo dos pacientes no contestaron cada uno a un ítem del cuestionario. 
Tabla 1. Características sociodemográficas y clínicas basales de los pacientes según gravedad de la HBP mediante I-PSS.

\begin{tabular}{|c|c|c|c|c|c|}
\hline & Leve & Moderada & Grave & Total & $\mathbf{p}$ \\
\hline Edad (años), media (DE) & $64,40(8,35)$ & $68,01(7,56)$ & $70,83(8,01)$ & $68,36(8,07)$ & $<0,01$ \\
\hline Nivel de estudios, \% (n) & & & & & $<0,01$ \\
\hline Universitarios & $13,9(33)$ & $11,2(106)$ & $8,5(44)$ & $10,8(183)$ & \\
\hline Secundarios (FP/BUP o similar) & $30,8(73)$ & $26,0(246)$ & $22,8(118)$ & 25,7 (437) & \\
\hline Primarios (EGB o similar) & $43,0(102)$ & $46,8(443)$ & 41,5 (215) & 44,7 (760) & \\
\hline Sin estudios & $11,8(28)$ & $15,3(145)$ & 24,9 (129) & $17,8(302)$ & \\
\hline Analfabeto & $0,4(1)$ & $0,2(2)$ & $2,3(12)$ & $0,9(15)$ & \\
\hline $\mathrm{NS} / \mathrm{NC}$ & & $0,4(4)$ & & $0,2(4)$ & \\
\hline TOTAL & $100(237)$ & $100(946)$ & $100(518)$ & $100(1.701)$ & \\
\hline $\begin{array}{l}\text { Tiempo desde el diagnóstico a la } \\
\text { basal (años), media (DE) }\end{array}$ & $2,12(3,17)$ & $2,28(3,26)$ & $2,66(3,63)$ & $2,37(3,37)$ & NS \\
\hline \multicolumn{6}{|c|}{ Reciben tratamiento para la HBP, \% (n) } \\
\hline No & $50,4(120)$ & 23,5 (224) & $21,3(111)$ & $26,6(455)$ & \\
\hline Si & 49,6 (118) & $76,5(729)$ & 78,7 (409) & $73,4(1.256)$ & $<0,01$ \\
\hline TOTAL & $100(238)$ & $100(953)$ & $100(520)$ & $100(1.711)$ & \\
\hline \multicolumn{6}{|l|}{ Enfermedades concomitantes, $\%(n){ }^{*}$} \\
\hline No & $28,5(68)$ & $22,5(215)$ & 20,7 (108) & 22,7 (391) & NS \\
\hline Sí & $71,5(171)$ & $77,5(742)$ & $79,3(415)$ & $77,3(1.328)$ & \\
\hline TOTAL & $100(239)$ & $100(957)$ & $100(523)$ & $100(1.719)$ & \\
\hline Hipertensión & $39,8(68)$ & $45,8(340)$ & $57,3(238)$ & 48,6 (646) & $<0,01$ \\
\hline Colesterol y/o triglicéridos & $32,7(56)$ & $31,3(232)$ & $33,0(137)$ & $32,0(425)$ & NS \\
\hline Enfermedades respiratorias & $11,7(20)$ & 19,7 (146) & $25,1(104)$ & $20,3(270)$ & $<0,01$ \\
\hline Diabetes & $14,6(25)$ & $19,4(144)$ & $22,2(92)$ & $19,7(261)$ & NS \\
\hline Enfermedades cardiovasculares & $11,1(19)$ & $16,8(125)$ & $20,5(85)$ & 17,2 (229) & $<0,05$ \\
\hline Otros & $65(111)$ & $58,1(429)$ & $52,1(215)$ & $57,5(755)$ & \\
\hline
\end{tabular}

* La suma de los subtotales de cada enfermedad no coincide con la muestra total que presentaba alguna enfermedad concomitante puesto que un mismo caso podía presentar más de un diagnóstico.

Tabla 2. Puntuaciones basales del I-PSS, item Bother Score y BII.

\begin{tabular}{lccccc}
\hline Cuestionario /Gravedad & Leve & Moderada & Grave & Total & p \\
\hline I-PSS, media (DE) & $5,39(1,48)$ & $13,96(3,10)$ & $25,03(3,90)$ & $16,13(7,28)$ & $<0,01$ \\
Bother Score, media (DE) & $1,73(1,03)$ & $3,12(1,02)$ & $4,42(0,89)$ & $3,32(1,31)$ & $<0,01$ \\
BII, media (DE) & $2,59(2,21)$ & $5,92(2,75)$ & $9,13(2,52)$ & $6,43(3,35)$ & $<0,01$ \\
\hline
\end{tabular}

\section{Validez transversal}

En general, la correlación de la puntuación del BII con el I-PSS $(\mathrm{r}=0,698)$ con el item de calidad de vida Bother Score $(r=0,682)$ y con el estado de salud percibido por el paciente $(r=0,66)$ fue moderada-alta y estadísticamente significativa $(p<0,01)$.

Las puntuaciones del BII difirieron de manera estadísticamente significativa $(\mathrm{p}<0,01)$ según la gravedad de la sintomatología, siendo más elevadas en los pacientes más graves.

Para comprobar que, el hecho de que los pacientes más graves padecían en mayor proporción enfermedades como hipertensión, enfermedades respiratorias o cardiovasculares, no repercutía en las puntuaciones del BII, se realizó un modelo de regresión lineal controlando el efecto de la gravedad. El resultado fue que la presencia de estas enfermedades concomitantes no era relevante en las respuestas de los pacientes al cuestionario BII $(\mathrm{p}=0,80 ; \mathrm{p}=147 ; \mathrm{p}=0,278)$, mostrándose solamente la gravedad significativa $(p<0,01)$.

Del mismo modo, las puntuaciones del BII fueron significativamente diferentes según la pregunta del estado de salud percibido por el paciente y según el estado de salud valorado por el médico $(p<0,01)$. Los pacientes que declararon un peor estado de salud obtuvieron puntuaciones mayores en el cuestionario BII (una peor calidad de vida) que los pacientes con un mejor estado de salud. 


\section{Validez longitudinal}

Los cambios en la CVRS se correlacionaron de manera moderada-alta con los cambios en el I-PSS $\mathrm{y}$ el item Bother Score ( $\mathrm{r}=0,61$ y 0,57) y de forma moderada con los cambios en la percepción del estado de salud del paciente $(r=-0,47$ y $-0,54)$ en la evaluación a los 6 meses de seguimiento.

Estos cambios en las puntuaciones en el BII también se relacionaron de forma estadísticamente significativa con los cambios percibidos por el médico en el estado de salud del paciente $(p<0,01)$.

\section{Fiabilidad}

La consistencia interna entre los itemes fue alta, con un coeficiente $\alpha$ de Cronbach global de 0,91 , al igual que la estabilidad de las puntuaciones entre las dos primeras visitas, a los 3 y a los 6 meses (fiabilidad test-retest), con un CCI de 0,87.

\section{Sensibilidad al cambio}

Los pacientes que declaraban que su estado de salud respecto a la visita basal prácticamente no había cambiado, mostraron un tamaño del efecto pequeño y cambios medios (DE) de 0,67
$(2,14)$ puntos en las puntuaciones del cuestionario. Del mismo modo, aquellos pacientes que consideraban que habian mejorado de forma moderada, presentaron un tamaño del efecto medio y cambios en las puntuaciones (DE) de 2,07 $(2,19)$ puntos. Finalmente, el tamaño del efecto mayor se dio en los pacientes que declararon estar mucho mejor o mucho peor, fueron los pacientes con las puntuaciones medias en el cuestionario más elevadas y que mostraron un cambio mayor en las puntuaciones (DE), de 4,02 $(2,96)$ puntos.

El tamaño del efecto de los pacientes que habían mejorado de forma moderada $(0,6)$, se consideró como la MDCI, es decir, el cambio más pequeño en la puntuación que los pacientes percibían como beneficioso era de 2 puntos (Tabla 3 ).

Los cambios detectados por el cuestionario difieren según la gravedad de la sintomatología, cuando mayor es la gravedad mayores cambios en la calidad de vida de los pacientes, así como una mayor MDCI. Para los pacientes graves un cambio mínimo en la puntuación del cuestionario de 3 puntos se percibe como una mejoría, mientras que en los pacientes leves esta diferencia es de 0,7 puntos (Tabla 4)

Tabla 3. Descripción de las puntuaciones del BII en la visita basal y a los 6 meses, media del cambio percibido y tamaño del efecto.

\begin{tabular}{lccccc}
\hline $\begin{array}{l}\text { Estado salud/puntuaciones } \\
\text { Media (DE) }\end{array}$ & Basal & $\mathbf{6 ~ M e s e s}$ & Diferencia & N & Tamaño Efecto \\
\hline Mucho mejor & $6,18(3,19)$ & $2,16(1,85)$ & $4,02(2,96)$ & 130 & 1,3 \\
Bastante mejor & $7,07(2,99)$ & $3,82(2,24)$ & $3,25(2,54)$ & 502 & 1,1 \\
Moderadamente mejor & $7,02(3,26)$ & $4,95(2,57)$ & $2,07(2,19)$ & 511 & 0,6 \\
Mas o menos igual & $5,05(3,44)$ & $4,38(3,00)$ & $0,67(2,14)$ & 432 & 0,2 \\
Moderadamente peor & $5,96(3,75)$ & $6,65(3,68)$ & $-0,69(0,16)$ & 49 & $-0,2$ \\
Bastante/Mucho peor & $7,45(2,98)$ & $10,64(2,01)$ & $-3,18(2,64)$ & 11 & $-1,1$ \\
TOTAL & $6,42(3,35)$ & $4,32(2,77)$ & $2,1(2,71)$ & 1635 & 0,6
\end{tabular}

Tabla 4. Descripción media del cambio percibido en las puntuaciones y tamaño del efecto para los pacientes según la gravedad.

\begin{tabular}{|c|c|c|c|c|c|c|}
\hline \multirow[t]{2}{*}{ Estado salud/puntuaciones } & \multicolumn{3}{|c|}{ Diferencia Media (DE) } & \multicolumn{3}{|c|}{ Tamaño efecto } \\
\hline & Leve & Moderada & Grave & Leve & Moderada & Grave \\
\hline Mucho mejor & $1,79(2,15)$ & $4,21(2,73)$ & $5,93(2,79)$ & 0,9 & 1,5 & 2,2 \\
\hline Bastante mejor & $1,41(2,39)$ & $3,00(2,28)$ & $4,17(2,61)$ & 0,6 & 1,2 & 1,9 \\
\hline Moderadamente mejor & $0,73(1,84)$ & $1,78(1,98)$ & $2,98(2,27)$ & 0,3 & 0,7 & 1,1 \\
\hline Mas o menos igual & $0,05(1,14)$ & $0,43(2,18)$ & $1,92(2,28)$ & 0,0 & 0,1 & 0,7 \\
\hline Moderadamente peor & $-3,25(2,99)$ & $-0,71(3,42)$ & $0,07(2,27)$ & $-3,4$ & $-0,2$ & 0,0 \\
\hline Bastante/Mucho peor & - & $-3,17(1,94)$ & $-3,20(3,56)$ & - & $-1,3$ & $-0,9$ \\
\hline TOTAL & $0,67(1,98)$ & $1,85(2,60)$ & $3,23(2,78)$ & 0,3 & 0,7 & 1,3 \\
\hline
\end{tabular}




\section{CVRS}

La calidad de vida evaluada en los pacientes del estudio mediante el cuestionario BII mostró una mejora significativa entre la visita basal y los 6 meses (menores puntuaciones) $(p<0,01)$. Se pasó de una puntuación basal media de 6,4 puntos a 4,3 puntos transcurridos 6 meses del inicio del estudio. La evolución de la calidad de vida fue significativa en cada uno de los grupos de gravedad $(\mathrm{p}<0,01)$, aunque como se observa en la Figura 2 la evolución era más notable en los pacientes con una mayor gravedad de los sintomas.

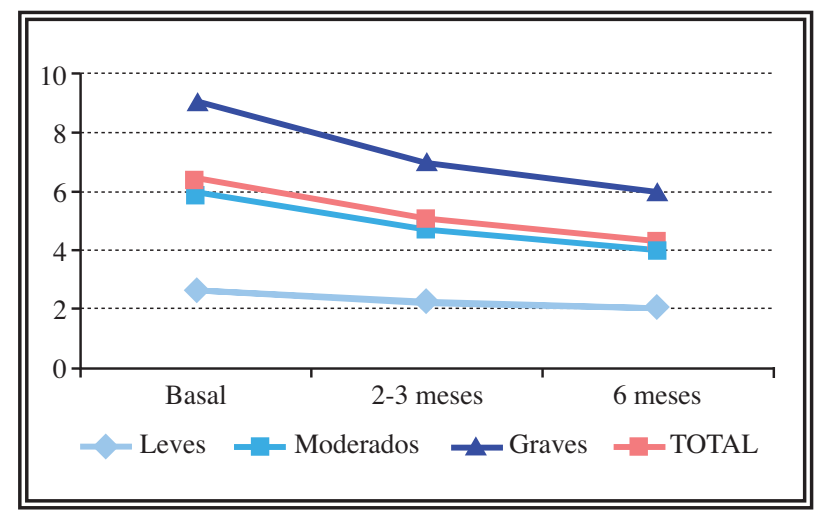

FIGURA 2. Evolución de la calidad de vida a lo largo del estudio según grupos de gravedad (I-PSS).

\section{DISCUSIÓN}

El presente estudio muestra que la versión española del cuestionario Benign Prostatic Hyperplasia Impact Index es útil para evaluar la CVRS en pacientes con HBP, con cualquier grado de gravedad ya que se muestra como una herramienta factible, válida, fiable y sensible a los cambios.

El cuestionario BII es de fácil comprensión, tal y como indica el elevado porcentaje de pacientes que lo respondieron en su totalidad $(99,9 \%)$.

La baja correlación entre las puntuaciones del BII y la edad de los pacientes discrepa con la elevada correlación que se encuentra al administrar el cuestionario I-PSS. Puede parecer contradictorio pero hay que puntualizar que el I-PSS es un cuestionario de síntomas objetivos y cuantificables y a mayor edad del paciente más graves son los síntomas físicos. En cambio, el cuestionario BII explora áreas de la CVRS, los pacientes responden a aspectos subjetivos que pueden estar influenciados por la propia percepción o por el significado y la repercusión que la enfermedad tiene para cada persona, independientemente de la edad que tenga.

En cambio, las puntuaciones del BII se correlacionaron de forma moderada-alta con la gravedad de la HBP, es decir, que a mayor gravedad de la enfermedad peor calidad de vida.

Este cuestionario mostró una buena capacidad para medir la CVRS al compararlo con el item Bother Score que evalúa el mismo concepto. Esta relación corrobora la hallada en anteriores estudios $^{20}$.

Se confirma pues esta propiedad también en población española con índices muy similares a los de la versión original del cuestionario ${ }^{17}$ ( $\mathrm{r}=6,698$ y $\mathrm{r}=0,77$ respectivamente) así como con las correlaciones de otros cuestionarios específicos para síntomas urinarios como el DAN-PSS-1 ya mencionado anteriormente $(\mathrm{r}=0,71)^{16,20}$

Esta herramienta confirma el significativo impacto de HBP en la calidad de vida de los pacientes tal y como ya destacaron anteriores investigaciones.

Un aspecto que podía enmascarar este impacto de la HBP en la CVRS era el hecho de que los pacientes más graves eran también los que presentaban mayor incidencia de algunas enfermedades concomitantes. Podría ser que la calidad de vida fuera peor en los graves por este motivo. Esta hipótesis quedó descartada ya que si se controlaba el factor gravedad, las puntuaciones del cuestionario BII no aumentaban en presencia de enfermedades concomitantes.

La consistencia interna del BII supera los estándares psicométricos situados en torno al 0,7028.

La correlación entre los ítemes de la versión española del BII resultó más elevada que en la versión original (alfa de Cronbach=0,91 y 0,79 respectivamente) y también superior a la correlación entre los ítemes del cuestionario DAN-PSS-1 (alfa de Cronbach=0,73).

La fiabilidad de las puntuaciones a lo largo del tiempo resultó ser buena, es decir, los cambios que se puedan producir en las puntuaciones del BII en dos momentos distintos son debidos al error aleatorio solo en una proporción ínfima. Generalmente se acepta un $\mathrm{CCI} \geq \mathrm{a} 0,90^{28}$, en este caso se obtuvo un coeficiente de 0,87 , prácticamente el mismo que se obtuvo en la versión original y mayor al obtenido en el DAN-PSS-1. 
En general, las correlaciones entre los cambios en la gravedad de la sintomatología y los cambios en las puntuaciones fue moderada-alta, mientras que éstas se correlacionaron de forma moderada con los cambios en la percepción de los pacientes de su estado de salud a lo largo del estudio.

La disminución de las puntuaciones a lo largo del estudio indica una mejoría en la CVRS, aspecto que concuerda con el grado de gravedad y la percepción del estado de salud de los pacientes, que también fueron mejorando durante el seguimiento.

Los mayores cambios en las puntuaciones se dieron en aquellos pacientes que declaraban cambios más grandes en su estado de salud, el mismo modo los cambios fueron moderados en aquellos pacientes que percibieron también cambios moderados. Esto indica que el BII es una herramienta sensible a los cambios que pueda haber en la CVRS relacionada con la HBP.

El tamaño del efecto de los pacientes que declaraban haber mejorado de forma moderada fue la puntuación media del cambio que se consideró como la MDCI.

Recientemente, el cuestionario BII ha sido utilizado en estudios sobre la efectividad de dutasteride, como el de Desgrandchamps $\mathrm{F}$ et al. $2006^{29}$. En este estudio se evaluó el impacto del fármaco no sólo en los sintomas físicos, sino también en la calidad de vida, utilizando como variables principales de resultados el I-PSS y el BII. La correlación obtenida entre las puntuaciones de ambos instrumentos fue estadísticamente significativa ( $<<0,001)$. La CVRS mejoró de forma significativa a los 6 meses de tratamiento con dutasteride.

Hakenberg O et al. $2001^{30}$ también utilizaron el cuestionario BII, entre otras medidas, para evaluar el grado de mejoría de los sintomas de la HBP en pacientes en los que se había practicado una resección transuretral de próstata. Concretamente el BII se administró antes y después de la intervención a los 3 y 6 meses. En todas las evaluaciones el BII mostró una mejoría estadísticamente significativa en sus puntuaciones, al igual que ocurrió con las puntuaciones del I-PSS.

La validación presentada en este estudio muestra que esta versión española del cuestiona- rio BII es un instrumento factible, válido, fiable y sensible a los cambios y por lo tanto disponemos de una herramienta válida para evaluar la CVRS de los pacientes con HBP en España, como ya se ha demostrado con anterioridad en validaciones en otros países.

Las recomendaciones internacionales ${ }^{12}$ asî como diversos estudios que las apoyan ${ }^{5,9}$ insisten en la importancia de una evaluación global del paciente con HBP. No sólo se deben evaluar los sintomas y manifestaciones clínicas sino también como éstos afectan en la calidad de vida de los afectados. En población española no existía, hasta el momento, ningún cuestionario específico que evaluara la calidad de vida en pacientes con HBP.

Con este estudio se consigue ajustar más la evaluación de los pacientes con HBP siguiendo estas recomendaciones, aportando un nuevo instrumento para la población española validado y específico para esta enfermedad.

Anexo: Esta investigación ha sido financiada por GlaxoSmithKline S.A.

\section{REFERENCIAS}

1. Roehrborn C. Reducing the risk of benign prostatic hyperplasia progression. Rev Urol. 2002;4 Suppl 5:S29-38.

2. Welsch G, Weinger K, Barry MJ. Quality-of-life impact of lower urinary tract symptom severity: results from the Health Professionals Follow-up Study. Urology 2000;59(2): 245-250.

3. Shabbir M, Mumataz FH. Benign prostatic hyperplasia. J R Soc Health. 2004;124(5):222-227.

4. Hellstrom MD. Benign prostatic hyperplasia, sexual function, and overall evaluation of the male patient. J Am Osteopath Assoc. 2004;104(2 Suppl 2):S5-10.

5. Barry MJ. Evaluation of symptoms and quality of life in men with benign prostatic hyperplasia. Urology. 2001;58(6 Supp1 1):25-32.

6. Alonso E, Sánchez $M^{a} D$, Fonz C, Fernández C, Campos M. Hiperplasia próstatica benigna: perspectivas de presente y de futuro. Revista de la sociedad madrileña de medicina de familia y comunitaria 2002;4(3):50-52.

7. Fitzpatrick J. The natural history of benign prostatic hyperplasia. BJU Int. 2006;97(Suppl 2):3-6.

8. Girman CJ, Epstein RS, Jacobsen SJ, Guess HA, Panser LA, Oesterling JE, et al. Natural history of prostatism: impact of urinary symptoms on quality of life in 2115 randomily selected community men. Urology. 1994;44(6):825831.

9. O'Leary MP. LUTS, ED, QOL: alphabet soup or real concerns to aging men?. Urology. 2000;56(5 Suppl 1):7-11.

10. Wright JG. Evaluating the outcome of treatment: shouldn't we be asking patients if they are better?. J Clin Epidemiol. 2000;53(6):549-553. 
11. Badia X, Carné X. La evaluación de la calidad de vida en el contexto del ensayo clínico. Med Clin (Barc). 1998;110 (14):550-556

12. Roehrborn CG. Focus on lower urinary tract symptoms: nomenclature, diagnosis, and treatment options: highlights from the 5 th international consultation on benign prostatic hyperplasia june 25-27, 2000, paris, france. Rev Urol. 2001;3(3):139-145.

13. Trueman P, Hood SC, Nayak US, Mrazek MF. Prevalence of lower urinary tract symptoms and self-reported diagnosed 'benign prostatic hyperplasia', and their effect on quality of life in a community-based survey of men in the UK. BJU Int. 1999;83(4):410-415.

14. Gómez Acebo A, Rodríguez Vallejo JM, Rodríguez Mora VI, García Alcázar I. Calidad de vida y sintomatología en la hiperplasia prostática benigna en población activa española. Med Clin (Barc) 2000;114(Suppl 3):81-89.

15. Suzuki H, Yano M, Awa Y, Nakatsu H, Egoshi K, Mikami K, et al. Clinical impact of tamsulosin on generic and symptom-specific quality of life for benign prostatic hyperplasia patients: using international prostate symptom score and Rand Medical Outcomes Study 36-item Health Survey. Int J Urol. 2006;13(9):1202-1206.

16. Hansen BJ, Flyer H, Brasso K, Schou J, Nordling J, Thorup $\mathrm{J}$ et al. Validation of the self-administered Danish Prostatic Symptom Score (DAN-PSS-I) system for use in benign prostatic hyperplasia. Br J Urol. 1995;76(4):451458.

17. Barry MJ, Fowler FJ Jr, O'Leary MP, Bruskewitz RC, Holtgrewe HL, Mebust WK. Measuring disease-specific health status in men with benign prostatic hyperplasia. Med Care. 1995;33(Suppl 4):AS145-155.

18. Homma Y, Tsukamoto T, Yasuda K, Ozono S, Yoshida M, Shinji M. Linguistic validation of japanese version of International Prostatic Symptom Score and BPH impact index. Nippon Hinyokika Gakkai Zasshi. 2002;93(6):669680.

19. Badia X, García-Losa M, Serra M, Carballido J, Herdman M. Symptom indices and quality of life questionnaires for use in benign prostatic hyperplasia. Dis Manage Health Outcomes. 1997;2(6):291-301.

20. Boyle P, Robertson C, Mazzetta C, Keech M, Hobbs R, Fourcade $\mathrm{R}$ et al. The relationship between lower urinary tract symptoms and health status: the UREPIK study. BJU Int. 2003;92(6):575-580.
21. Vela Navarrete R, Martín Moreno JM, Calahorra FJ, Damián Moreno J, Hernández Coronado A, Boyle P. Cultural and linguistic validation, in Spanish, of the International Prostatic Symptoms Scale (I-PSS). Actas Urol Esp. 1994;18 (8):841-847.

22. Barry MJ, Fowler FJ, Jr., O'Leary MP, Bruskewitz RC, Holtgrewe HL, Mebust WK et al. The American Urological Association Symptom Index for benign prostatic hyperplasia. The Measurement Committee of the American Urological Association. J Urol 1992;148(5):1549-1557.

23. Hays RD, Anderson R, Revicki D. Psychometric considerations in evaluating health-related quality of life measures. Qual Life Res. 1993;2(6):441-449.

24. Prieto L, Lamarca R, Casado A. La evaluación de la fiabilidad en las observaciones clínicas: el coeficiente de correlación intraclase. Med Clin (Barc). 1998;110(4):142-145.

25. Fleiss JL. The design and analysis of clinical experiments. New York: John Wiley \& Sons, Inc.; 1986.

26. Cohen J. Statistical power analysis for the behavioural sciences. New Yersey: Lawrence Eribaum Associates Inc.; 1988.

27. Badia X, Lizán L. Estudios de calidad de vida. En: Martín A, Can JF. Atención Primaria: conceptos, organización y práctica clínica. Vol I. Madrid: Elsevier España; 2003; p. 250-261.

28. Hays RD, Anderson R, Revicki D. Psychometric considerations in evaluating health-related quality of life measures. Qual Life Res. 1993;2(6):441-449.

29. Desgrandchamps F, Droupy S, Irani J, Saussine C, Comenducci A. Effect os dutasteride on the symptoms of benign prostatic hyperplasia, and patient quality of life and discomfort, in clinical practice. BJU Int. 2006;98(1): 83-88.

30. Hakenberg OW, Helke C, Manseck A, Wirth MP. Is there a relationship between the amount of tissue removed at trasurethral resction of the prostate and clinical improvement in benign prostatic hyperplasia?. Eur Urol. 2001;39(4): 412-417.

Correspondencia autor: Dr. X Badía Llach

Health Economics \& Outcomes Research. IMS Health

E-mail autor: xbadia@es.imshealth.com

Información artículo: Original - Hiperplasia benigna de próstata

Trabajo recibido: junio 2007

Trabajo aceptado: julio 2007 